\title{
Assessment of subclinical left ventricle myocardial dysfunction using global myocardial work in type 2 diabetes mellitus patients with preserved left ventricle ejection fraction
}

\author{
Tao Wang ${ }^{1}$, Li Li ${ }^{2}$, Jun Huang ${ }^{3^{*}}$ and Li Fan ${ }^{3}$
}

\begin{abstract}
Background: The purpose of this study was to assess subclinical left ventricle (LV) myocardial dysfunction using global myocardial work (MW) in type 2 diabetes mellitus (T2DM) patients with preserved left ventricle ejection fraction (LVEF).

Methods: Sixty T2DM patients and 60 normal controls were enrolled in the study. Apical 4-, 3- and 2-chamber views were acquired by two-dimensional echocardiography. Peak systolic myocardial global longitudinal strain (GLS), global myocardial work index (GWI), global constructive work (GCW), global wasted work (GWW), and myocardial work efficiency (GWE) were determined by speckle-tracking echocardiography (STE).

Results: The GLS values in the T2DM patients were significantly lower than those in normal controls $(p<0.001)$. The GWW in T2DM patients was significantly greater than that in normal controls, while GWI, GCW and GWE was significantly lower $(p<0.001)$. Receiver operating characteristic $(R O C)$ analysis showed there were no significant different difference between GWW, GWE and GLS in the area under the curves (AUCS). In T2DM patients, fasting plasma glucose was positively correlated with GWW but negatively correlated with GWE, and GLS was negatively correlated with GWI and GCW.

Conclusion: From the research, we found that global MW as new technique could detect the subclinical LV myocardial dysfunction and confirm that the impaired LV function in T2DM patients with preserved LV systolic function.
\end{abstract}

Keywords: Myocardial work, Global longitudinal strain, Subclinical LV dysfunction, Type 2 diabetes mellitus

\section{Background}

The incidence of type 2 diabetes mellitus (T2DM) has increased worldwide in recent years. The development of coronary artery disease induced by T2DM has also increased. In the past few years, cardiovascular disease has become the major cause of morbidity and mortality

\footnotetext{
*Correspondence: 305669112@qq.com

${ }^{3}$ Department of Echocardiography, The Affiliated Changzhou No. 2

People's Hospital with Nanjing Medical University, Changzhou 213003, China

Full list of author information is available at the end of the article
}

for T2DM patients [1]. The pathological changes of diabetic-induced cardiomyopathy mainly include cardiomyocyte apoptosis, myocardial fibrosis and necrosis, ultimately leading to systolic and diastolic cardiac dysfunction and heart failure [2,3]. Therefore, to protect cardiac function, early detection and intervention of the function of the left ventricle (LV) is essential to the prevention and management of diabetic cardiomyopathy.

Many techniques can be used to assess cardiac function, such as cardiovascular magnetic resonance T1 mapping [4], Tc-99 m MIBI gated single-photon



(c) The Author(s) 2022. Open Access This article is licensed under a Creative Commons Attribution 4.0 International License, which permits use, sharing, adaptation, distribution and reproduction in any medium or format, as long as you give appropriate credit to the original author(s) and the source, provide a link to the Creative Commons licence, and indicate if changes were made. The images or other third party material in this article are included in the article's Creative Commons licence, unless indicated otherwise in a credit line to the material. If material is not included in the article's Creative Commons licence and your intended use is not permitted by statutory regulation or exceeds the permitted use, you will need to obtain permission directly from the copyright holder. To view a copy of this licence, visit http://creativecommons.org/licenses/by/4.0/. The Creative Commons Public Domain Dedication waiver (http://creativeco mmons.org/publicdomain/zero/1.0/) applies to the data made available in this article, unless otherwise stated in a credit line to the data. 
emission-computed tomography myocardial perfusion imaging [5], tissue Doppler strain echocardiography [6], and two-dimensional speckle tracking echocardiography (2D-STE) [7, 8]. However, these techniques all have limitations. Like angle dependence in tissue Doppler strain echocardiography, long-term examination in cardiac magnetic resonance imaging and the radioactivity of cardiac radionuclide imaging, 2D-STE has a lower signal-to-noise ratio, weak acoustic windows, and through-plane motion artefacts $[9,10]$. However, 2D-STE has become the leading reliable diagnostic technique for the assessment of cardiac function because of its noninvasiveness, convenience, and repeatability. In addition, our group previously reported that LV longitudinal myocardial function detected by longitudinal strain and rotation in T2DM patients with preserved LV ejection fraction (LVEF) was impaired when compared with that in healthy subjects [11]. However, global myocardial work $(\mathrm{MW})$ is a new parameter for 2D-STE that takes into account deformation as well as afterload through interpretation of strain in relation to dynamic noninvasive LV pressure, potentially offering incremental value to myocardial function assessment.

The aim of the research was to explore the incremental value of global MW in the detection of subclinical LV myocardial dysfunction in T2DM patients with preserved LV systolic function.

\section{Subjects and methods \\ Ethical statement}

The study conducted according to the Principles of the Declaration of Helsinki and was approved by the human research and ethics committee of the affiliated Changzhou No. 2 people's hospital with Nanjing Medical University. All of the patients had completed the informed consent forms.

\section{Study population}

Sixty T2DM patients (not well-treated, and it means poor blood glucose control in these T2DM patients before hospitalization) and 60 normal controls of similar age and sex were enrolled for this study and recruited from the hospital. The inclusion criteria for T2DM patients were clinically confirmed in accordance with the World Health Organization criteria [12] and without any history of heart disease (congenital heart disease, coronary artery disease, arterial hypertension, myocardial infarction, cardiomyopathy, valvular disease, atrial fibrillation, thyroid disease, neoplastic disease, or kidney failure), and also without obesity and dyslipidemia. All enrolled subjects were performed with coronary CT or coronary angiography to confirm that they have no coronary artery disease. All the T2DM patients had a LVEF $>55 \%$. Normal controls were recruited from the physical examination in the hospital. In the normal controls, all of the physical and laboratory examination tests for cardiac function, electrocardiogram (ECG), and echocardiography were normal.

\section{Anthropometric and biochemistry}

The sex, age, height, body weight, heart rate (HR) and blood pressure (systolic blood pressure: SBP, diastolic blood pressure: DBP) of all subjects were recorded at the time of the echocardiography examination. Fasting plasma glucose, glycated haemoglobin (HbA1c), total cholesterol $(\mathrm{TCH})$, triglyceride (TG), high density lipoprotein (HDL) and low density lipoprotein (LDL) were measured when the patients were in hospital.

\section{Conventional 2D Doppler echocardiography}

Patients underwent conventional 2D transthoracic Doppler echocardiography with a Vivid E9 equipped with an M5S 3.5-5 MHz transducer (GE Vingmed Ultrasound, Horten, Norway) by an experienced cardiologist. All of the patients were connected to ECG leads. Apical 4-chamber, 2-chamber, and long-axis views of three consecutive cycles with a standard high frame rate $\left(>45 \mathrm{~s}^{-1}\right)$ were stored for offline analysis. The examination of echocardiography and the measurement of fasting plasma glucose, HbA1c, TCH, TG, HDL and LDL were performed in the same day.

Echocardiographic parameters of left atrial diameter (LAd), interventricular septum thickness (IVSd), LV posterior wall thickness (LVPWd) and LV diameter (LVDd) in the end-diastole period, the peak early (E) and late (A) diastolic mitral annular velocities, LV end-diastole volume (LVEDV), LV end-systole volume (LVESV) and LVEF (modified biplane Simpson's method), and the peak early $\left(\mathrm{e}^{\prime}\right)$ and late $\left(\mathrm{a}^{\prime}\right)$ diastolic annular velocities (obtained by averaging the values at the septum and lateral wall by tissue Doppler imaging: TDI) were measured during the examination.

\section{Two-dimensional STE}

Global longitudinal strain and MW were measured by EchoPAC software (EchoPAC Version: 203, GE Vingmed Ultrasound, Norway).

First, the aortic valve closure time was identified from the event timing of the aortic valve spectrum. Then, APLAX, A4C and A2C were used to analyse the apical long-axis, four-chamber and two-chamber views. The LV myocardium was divided into 18 segments, and the global longitudinal strain (GLS) was automatically measured by the software (Fig. 1A and B).

MW and related indices were calculated using a combination of echocardiographic strain data and a 

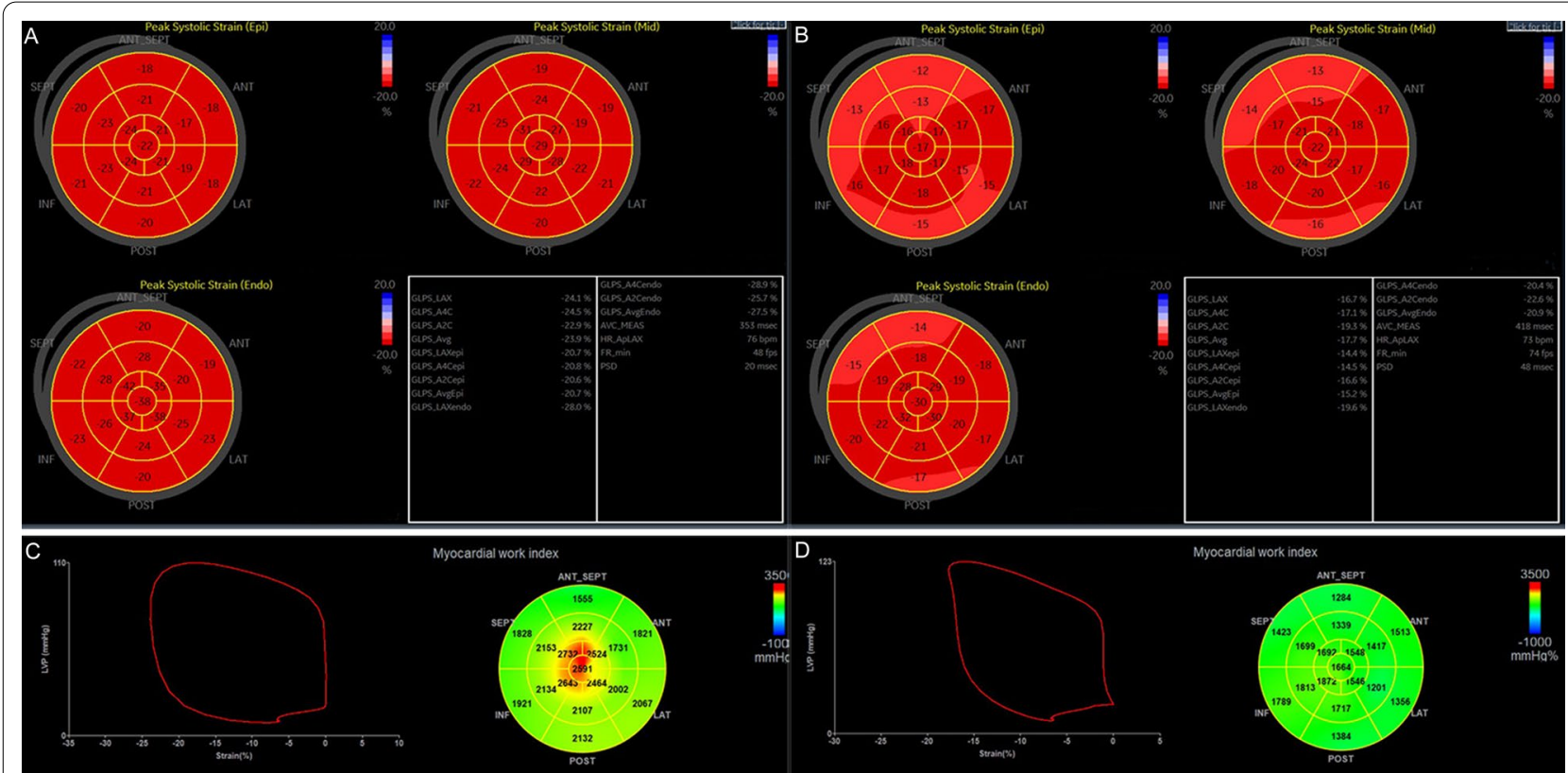

Myocardial work index
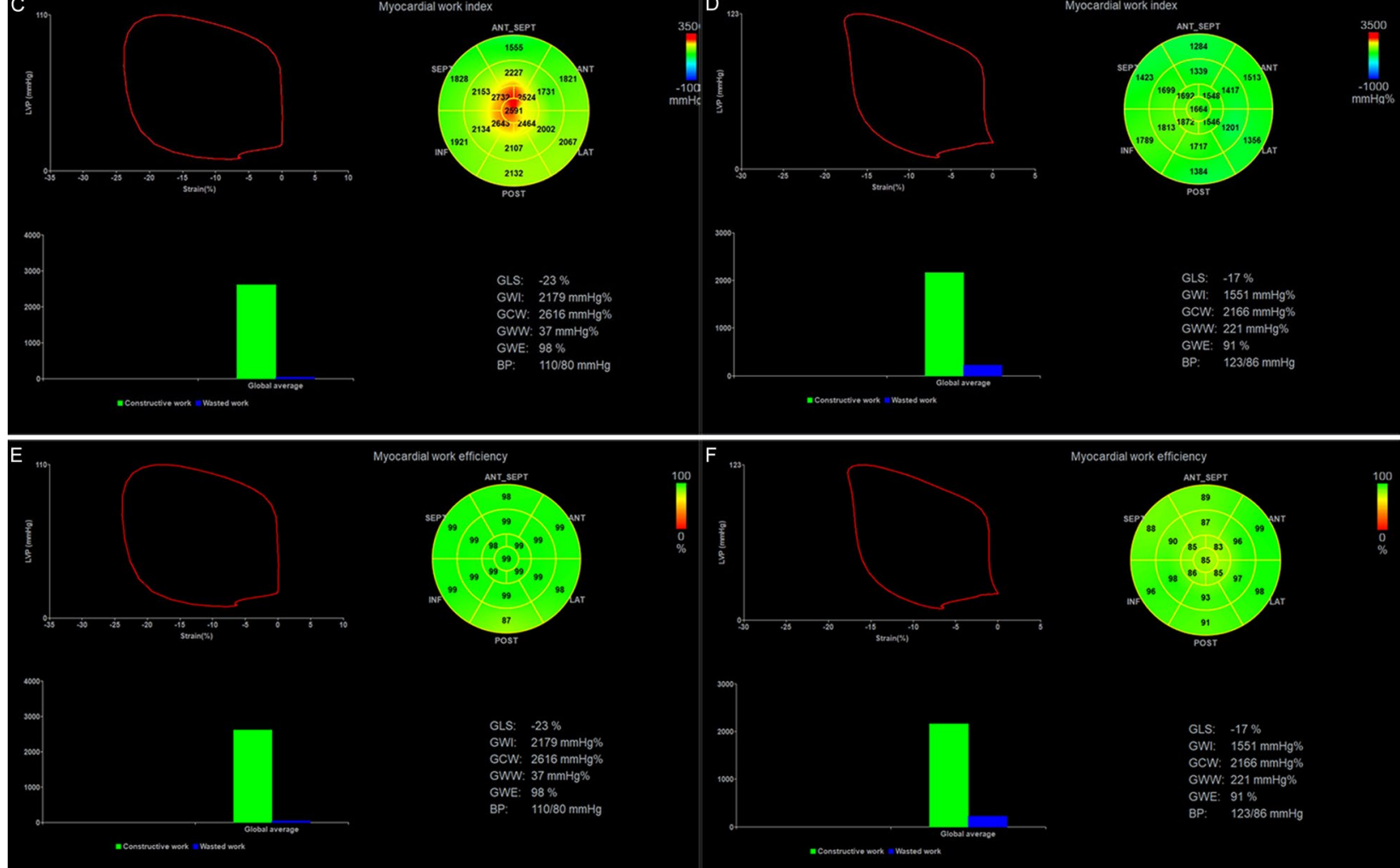

Myocardial work efficiency

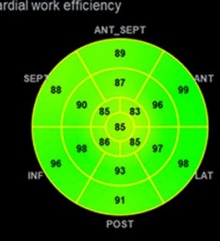

Fig. 1 The bull's eyes of the peak systolic myocardial longitudinal strain of normal controls and T2DM patients (A and B). global myocardial work of normal controls and T2DM patients $(\mathbf{C}-\mathbf{F})$

noninvasively estimated LV pressure curve. Blood pressure was input into the software, and "Advanced" was clicked. The software constructed a non-invasive LV pressure curve, then, MW and related indices were calculated using a combination of echocardiographic strain data and a noninvasively estimated LV pressure curve [13, 14]: (1) GWI: global myocardial work index, means the area within the global LV pressure-strain loop, calculated from mitral valve closure to mitral valve opening. (2) GCW: global constructive work, means work performed by the LV contributing to LV ejection during systole, estimated the work by the LV segments shortening during systole plus lengthening during isovolumic relaxation. (3) GWW: global wasted work, means work performed by the LV that does not contribute to LV ejection, estimated the work by the LV segments lengthening during systole plus shortening during the isovolumic relaxation phase. (4) GWE: myocardial work efficiency, GWE $=\mathrm{GCW} /(\mathrm{GCW}+\mathrm{GWW})$ (Fig. $1 \mathrm{C}-\mathrm{F}$ ). 


\section{Statistical analysis}

All data analyses were performed using SPSS 25.0 software (SPSS, Chicago, IL, USA). The Shapiro-Wilk test or Kolmogorov-Smirnov test was used to detect the normality of all values. Differences between the T2DM patients and normal controls were compared with an independent Student's t-test because the data distribution was normal. For variables with a nonnormal distribution, the nonparametric Mann-Whitney test was used. We defined GLS and global MW values in the normal controls as the normal state and considered the values of T2DM patients to be abnormal. These values in T2DM patients were determined from receiver operating characteristic (ROC) curve analysis by MedCalc software. Youden's index was selected as the cut-off point that can give the best composite of specificity and sensitivity. Correlations among LVEF, fasting plasma glucose, HbA1c and GLS and global MW values were tested using Pearson or Spearman correlation tests as appropriate. The categorical variables are presented as frequencies and percentages. Data are presented as the mean \pm standard deviation (SD) if normally distributed, and as medians and interquartile range boundaries otherwise. A p-value $<0.05$ was considered significant in all tests.

\section{Reproducibility and repeatability}

Intraobserver and interobserver variability for GLS and global MW values were determined by repeating measurements in 25 patients randomly selected from among all enrolled patients.

\section{Results}

\section{Patient characteristics}

A total of 120 patients met the baseline inclusion criteria. Nineteen patients were excluded from the strain and MW analyses because of inadequate image quality $(n=12)$, tachycardia $(n=7)$ or irregular heartbeat $(n=3)$. A total of 98 patients were therefore evaluated in the study and were initially divided into two groups: normal controls $(\mathrm{n}=50$, mean age, $50.38 \pm 15.27$ years, 25 men $)$ and T2DM patients $(\mathrm{n}=48$, mean age, $53.79 \pm 11.24$ years, 25 men).

\section{Comparison of the patient characteristics and conventional echocardiographic parameters of normal controls and T2DM patients (Table 1)}

The values of SBP, fasting plasma glucose and HbA1c were significantly higher in the T2DM patients than in normal controls $(p<0.05)$. There were no significant differences in age, BMI, HR, sex, DBP, TCH, TG, HDL and
LDL between the normal controls and T2DM patients $(\mathrm{P}>0.05)$.

The values of LAd, IVSd, LVPWd, $\mathrm{a}^{\prime}$ and $\mathrm{E} / \mathrm{e}^{\prime}$ in the T2DM patients were significantly higher than those in normal controls $(\mathrm{p}<0.05)$, however, the LVEF, $\mathrm{E}$ and $\mathrm{e}^{\prime}$ values in the T2DM patients were significantly lower than those in normal controls $(\mathrm{p}<0.05)$. There were no significant differences in levels of LVDd, LVEDV, LVESV, $\mathrm{A}$ or E/A between the normal controls and T2DM patients $(\mathrm{p}>0.05)$.

\section{GLS and global MW in normal controls and T2DM patients (Table 2 and Fig. 2)}

The values of LV GLS in T2DM patients were significantly lower than those in normal controls $(\mathrm{p}<0.001)$. The GWW value was significantly higher in T2DM patients than in normal controls, however, the GWI, GCW and GWE value was significantly lower in T2DM patients than in normal controls $(\mathrm{p}<0.001)$.

\section{ROC analysis (Table 3 and Fig. 3)}

ROC curve analysis was used to discriminate whether global MW parameters and GLS were able to predict subclinical LV myocardial dysfunction in T2DM patients. According to the ROC analysis, the area under the curve (AUC) of GLS was 0.840 , the cut-off value was $-21.1 \%$, with a sensitivity of $75 \%$ and specificity of $80 \%$. The AUC of GWI was 0.689 , the cut-off value was $2024 \mathrm{mmHg} \%$, with a sensitivity of $50 \%$ and specificity of $82 \%$. The AUC of GCW was 0.683 , the cut-off value was $2467 \mathrm{mmHg} \%$, with a sensitivity of $58 \%$ and specificity of $74 \%$. The AUC of GWW was 0.734 , the cut-off value was $46 \mathrm{mmHg} \%$, with a sensitivity of $96 \%$ and specificity of $46 \%$, and the AUC of GWE was 0.784 , the cut-off value was $97 \%$, with a sensitivity of $94 \%$ and specificity of $54 \%$. The comparation of AUCs showed that there was no significant difference between GWW, GWE and GLS ( $p>0.05)$.

\section{Correlation tests among LVEF, fasting plasma glucose, HbA1c, GLS and MW in T2DM patients (Table 4 and Figs. 4 and 5) \\ In T2DM patients, fasting plasma glucose was positively correlated with GWW but negatively correlated with GWE. GLS was negatively correlated with GWI and GCW.}

\section{Intra- and interobserver variability (Table 5)}

Intra- and interobserver variabilities were assessed via the intraclass correlation coefficients (ICCs) for the measurements. GLS and global MW parameters showed excellent intra- and interobserver correlations, with ICC values $>0.85$. 
Table 1 Baseline clinical characteristics, conventional two-dimensional echocardiographic parameters between T2DM patients and normal subjects

\begin{tabular}{|c|c|c|c|}
\hline Variable & Normal (50) & T2DM (48) & p-value \\
\hline \multicolumn{4}{|l|}{ Clinical } \\
\hline Age (yrs) & $50.38 \pm 15.27$ & $53.79 \pm 11.24$ & 0.212 \\
\hline Male (\%) & $25(50)$ & $25(52)$ & 0.846 \\
\hline $\mathrm{BMI}\left(\mathrm{kg} / \mathrm{m}^{2}\right)$ & $22.92 \pm 1.17$ & $22.68 \pm 1.44$ & 0.358 \\
\hline Heart Rate (bpm) & $73.72 \pm 10.74$ & $76.25 \pm 9.50$ & 0.221 \\
\hline $\mathrm{SBP}(\mathrm{mmHg})$ & $121.94 \pm 11.60$ & $127.15 \pm 12.91$ & 0.038 \\
\hline $\mathrm{DBP}(\mathrm{mmHg})$ & $76.78 \pm 8.33$ & $77.79 \pm 8.99$ & 0.565 \\
\hline Fasting plasma glucose (mmol/L) & $4.82 \pm 0.63$ & $13.28 \pm 4.36$ & $<0.001$ \\
\hline $\mathrm{HbA1c}(\%)$ & $5.02 \pm 0.62$ & $10.13 \pm 2.15$ & $<0.001$ \\
\hline $\mathrm{TCH}(\mathrm{mmol} / \mathrm{L})$ & $3.91 \pm 0.64$ & $3.99 \pm 0.77$ & 0.585 \\
\hline $\mathrm{TG}(\mathrm{mmol} / \mathrm{L})$ & $1.10 \pm 0.30$ & $1.19 \pm 0.60$ & 0.365 \\
\hline $\mathrm{HDL}(\mathrm{mmol} / \mathrm{L})$ & $1.27 \pm 0.29$ & $1.19 \pm 0.36$ & 0.272 \\
\hline LDL (mmol/L) & $2.06 \pm 0.50$ & $2.27 \pm 0.62$ & 0.060 \\
\hline \multicolumn{4}{|c|}{ Medical treatment in hospitalization (\%) } \\
\hline Diet treatment & $0(0)$ & $0(0)$ & \\
\hline Oral drug & $0(0)$ & $12(25)$ & \\
\hline Insulin & $0(0)$ & $26(54)$ & \\
\hline Insulin + Oral drug & $0(0)$ & $10(21)$ & \\
\hline \multicolumn{4}{|l|}{ Echocardiography } \\
\hline $\mathrm{LAd}(\mathrm{mm})$ & $33.50 \pm 3.09$ & $35.46 \pm 3.91$ & 0.007 \\
\hline IVSd (mm) & $8.85 \pm 0.87$ & $9.35 \pm 1.23$ & 0.021 \\
\hline LVPWd (mm) & $8.54 \pm 0.95$ & $9.04 \pm 1.15$ & 0.021 \\
\hline LVDd (mm) & $46.30 \pm 2.52$ & $46.96 \pm 3.68$ & 0.302 \\
\hline LVEDV (ml) & $74.54 \pm 13.47$ & $72.52 \pm 14.07$ & 0.470 \\
\hline LVESV (ml) & $25.80 \pm 6.32$ & $27.44 \pm 5.61$ & 0.179 \\
\hline LVEF (\%) & $65.59 \pm 4.01$ & $62.06 \pm 4.87$ & $<0.001$ \\
\hline$E(\mathrm{~m} / \mathrm{s})$ & $0.88 \pm 0.13$ & $0.79 \pm 0.13$ & $<0.001$ \\
\hline$A(m / s)$ & $0.68 \pm 0.14$ & $0.69 \pm 0.19$ & 0.924 \\
\hline$E / A$ & $1.34 \pm 0.30$ & $1.22 \pm 0.34$ & 0.079 \\
\hline$e^{\prime}(m / s)$ & $0.13 \pm 0.03$ & $0.09 \pm 0.02$ & $<0.001$ \\
\hline$a^{\prime}(m / s)$ & $0.09 \pm 0.02$ & $0.10 \pm 0.02$ & $<0.001$ \\
\hline$E / e^{\prime}$ & $7.26 \pm 1.60$ & $10.35 \pm 2.55$ & $<0.001$ \\
\hline
\end{tabular}

$B M /$ body mass index, $S B P$ systolic blood pressure, $D B P$ diastolic blood pressure, $T C H$ total cholesterol, $T G$ triglyceride, $H D L$ high density lipoprotein, $L D L$ low density lipoprotein, $L A D$ left atrial diameter, IVSD interventricular septal thickness in end-diastolic period, LVPWD left ventricular posterior wall thickness in end-diastolic period, LVDD left ventricular diameter in end-diastolic period, LVEDV left ventricular end-diastolic volume, LVESV left ventricular end-systolic volume, LVEF left ventricular ejection fraction, $E$ peak velocity during early diastole of anterior mitral valve, $A$ peak velocity during late diastole of anterior mitral valve, $e^{\prime}$ peak early diastolic annular velocities using TDI, $a^{\prime}$ peak late diastolic annular velocities using TDI

\section{Discussion}

The main finding of the study was that subclinical LV myocardial dysfunction was detected by global MW in T2DM patients with preserved LV systolic function.

Cardiac impairment by T2DM includes microvascular impairment, metabolic disturbance, subcellular component abnormalities, cardiac autonomic dysfunction, and a maladaptive immune response. Eventually, functional and structural changes in myocardium without coronary artery disease are caused by T2DM, and this is called diabetic cardiomyopathy [15]. Diabetic cardiomyopathy may first induce diastolic dysfunction and then systolic dysfunction. Finally, clinical heart failure may occur. With the development of imaging examinations, the detection of cardiac function has become increasingly easy. Echocardiography for measuring MW is considered the newest tool for the assessment of LV systolic dysfunction. Billig et al. [16] evaluated left and right ventricular structure, function and myocardial work by transoesophageal echocardiography (TEE) in swine and found that myocardial contractility and mechanics could be reliably evaluated with noninvasive GWI derived from 
Table 2 GLS and Myocardial work between T2DM patients and normal subjects

\begin{tabular}{lllr}
\hline Variable & Normal (50) & T2DM (48) & p-value \\
\hline GLS (\%) & $-22.54 \pm 1.72$ & $-19.66 \pm 2.25$ & $<0.001$ \\
MW & & & \\
GWI (mmHg\%) & $2282.82 \pm 286.11$ & $2046.40 \pm 321.22$ & $<0.001$ \\
GCW (mmHg\%) & $2659.68 \pm 322.20$ & $2417.60 \pm 359.56$ & 0.001 \\
GWW (mmHg\%) & $64.00(46.00-90.50)$ & $86.00(61.25-121.75)$ & 0.002 \\
GWE (\%) & $97.00(96.00-98.00)$ & $96.00(94.25-97.00)$ & 0.001 \\
\hline
\end{tabular}

GLS global longitudinal strain, $M W$ myocardial work, GWI global myocardial work index, GCW global constructive myocardial work, GWW global wasted myocardial work, GWE global myocardial work efficiency

echocardiography without additional invasive measures. Galli et al. [17] provided reference values for GWI, GCW, GWW and GWE in a group of healthy volunteers controlling for age and sex and found that assessment of MW was feasible in normal subjects. The presented referral ranges of GWI, GCW, GWW and GWE were not affected by age. According to previous studies, MW has been reported in many diseases, including coronary heart disease [14], hypertension, cardiac resynchronization therapy (CRT), percutaneous coronary intervention (PCI) [18], aortic stenosis [19], transcatheter aortic valve replacement (TAVR) [20], dilated cardiomyopathy [21], hypertrophic cardiomyopathy [22], chronic kidney disease [23], and cardiac amyloidosis [24]. However, there has been little research about LV systolic function in T2DM patients with MW.

Liu et al. [25] examined the prognostic value of GLS in T2DM patients and found that in T2DM patients without any history of cardiovascular disease, impaired GLS was associated with cardiovascular events. Our research was consistent with this research, and we found that LV GLS was decreased in the T2DM patients compared with normal controls. MW analysis showed that the GWW value was significantly higher in T2DM patients than in normal controls and that the GWI, GCW and GWE value was significantly lower in T2DM patients than in normal controls. The results may indicate that subclinical LV myocardial dysfunction was present in T2DM patients, although LV systolic function was normal. We considered that the results were related to the pathological changes of the LV myocardium in T2DM. In T2DM, persistent hyperglycaemia causes molecular and metabolic changes in cardiomyocytes and damages the coronary microvasculature. Hypoxia of cardiomyocytes and ischaemia results in myocardial hypertrophy, perivascular and fibrosis, LV stiffness, and systolic and diastole dysfunction in T2DM [15]. Normal myocardial fibres consist of longitudinal and circumferential myocardial fibres. Almost $70 \%$ of the myocardial fibre is longitudinal,
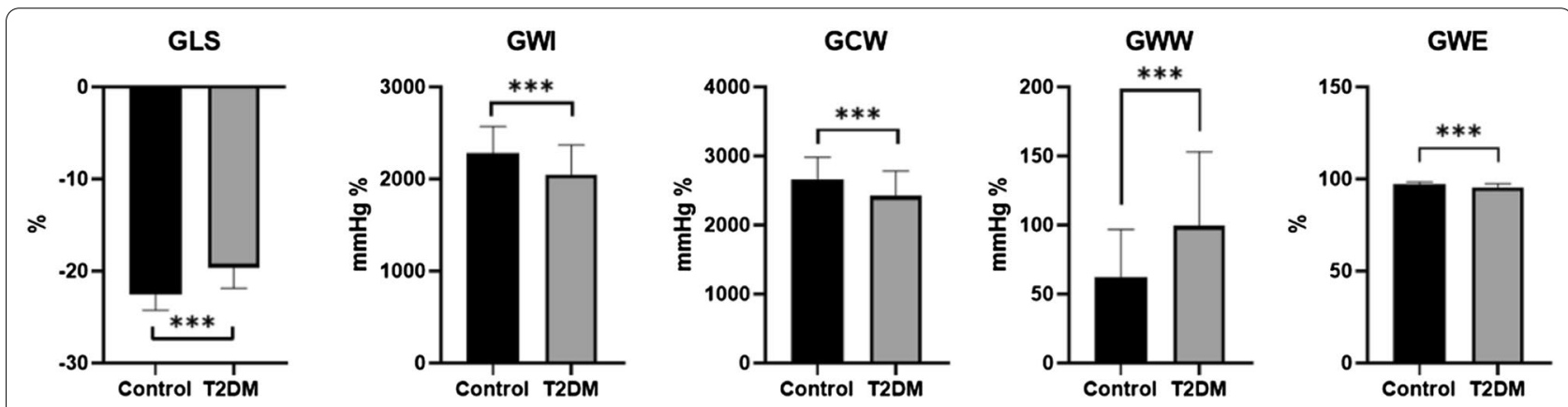

Fig. 2 GLS, GWI, GCW, GWW and GWE between normal controls and T2DM patients ( ${ }^{* * *}$ means $p<0.001$ )

Table 3 Receiver operating characteristic curve analysis for the detection LV dysfunction of T2DM patients

\begin{tabular}{llllll}
\hline Variable & GLS (\%) & GWI (mmHg\%) & GCW (mmHg\%) & GWW (mmHg\%) & GWE (\%) \\
\hline AUC (SE) & 0.840 & $0.689^{*}$ & $0.683^{*}$ & 0.734 & 0.784 \\
AUC (95\%Cl) & $0.753-0.907$ & $0.588-0.779$ & $0.581-0.773$ & $0.636-0.819$ & $0.689-0.861$ \\
Cut-off value & -21.1 & 2024 & 2467 & 46 & 97 \\
Sensitivity & 75 & 50 & 58 & 96 & 94 \\
Specificity & 80 & 82 & 74 & 46 & 54 \\
Youden index & 0.5500 & 0.3200 & 0.3233 & 0.4183 & 0.4775 \\
\hline
\end{tabular}

${ }^{*}$ Significantly different $(p<0.05)$ when global MW values compared with the GLS 


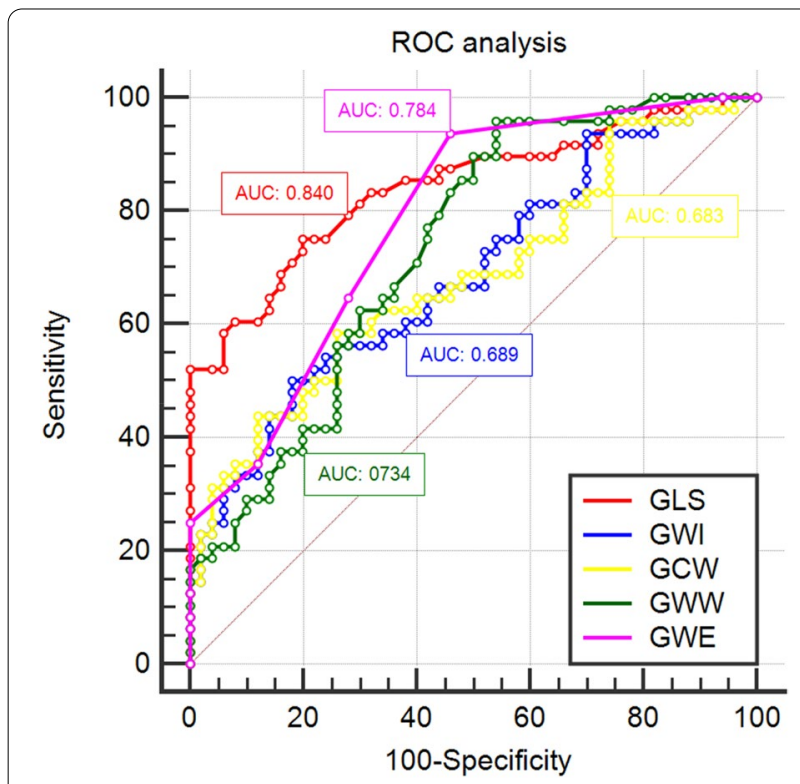

Fig. 3 ROC analysis for detecting the accuracy of subclinical LV dysfunction in T2DM patients and $30 \%$ is circumferential. If cardiomyocyte apoptosis, myocardial fibrosis and necrosis occur in T2DM, the sequence of the myocardium may change, eventually leading to damage to myocardial systolic function.

ROC analysis showed that GWW and GWE had the same diagnostic efficacy with GLS. The results may indicate global MW can evaluate the subclinical LV dysfunction in T2DM patients accurately.

In T2DM patients, fasting plasma glucose was positively correlated with GWW but negatively correlated with GWE, and GLS was negatively correlated with GWI and GCW. However, the correlations were not strong (0.3-0.6) and they could not accurately predict the subclinical LV myocardial dysfunction in T2DM patients.

\section{Conclusion}

From the research, we found that global MW as new technique could detect the subclinical LV myocardial dysfunction and confirm that the impaired LV function in T2DM patients with preserved LV systolic function.

Table 4 Correlation tests among fasting plasma glucose, LVEF, HbA1c, GLS and global MW in T2DM patients

\begin{tabular}{|c|c|c|c|c|c|c|c|c|}
\hline \multirow[t]{2}{*}{ Variable } & \multicolumn{2}{|l|}{ GWI } & \multicolumn{2}{|l|}{ GCW } & \multicolumn{2}{|l|}{ GWW } & \multicolumn{2}{|l|}{ GWE } \\
\hline & $r$ & $p$-value & $r$ & $p$-value & $r$ & $p$-value & $r$ & $p$-value \\
\hline $\begin{array}{l}\text { Fasting plasma } \\
\text { glucose }\end{array}$ & -0.005 & 0.971 & 0.059 & 0.689 & 0.410 & 0.004 & -0.392 & 0.006 \\
\hline $\mathrm{HbA1c}$ & -0.122 & 0.409 & -0.091 & 0.539 & 0.224 & 0.125 & -0.174 & 0.238 \\
\hline LVEF & 0.235 & 0.109 & 0.150 & 0.310 & -0.141 & 0.340 & 0.204 & 0.165 \\
\hline GLS & -0.343 & 0.017 & -0.516 & $<0.001$ & -0.059 & 0.692 & -0.138 & 0.351 \\
\hline
\end{tabular}
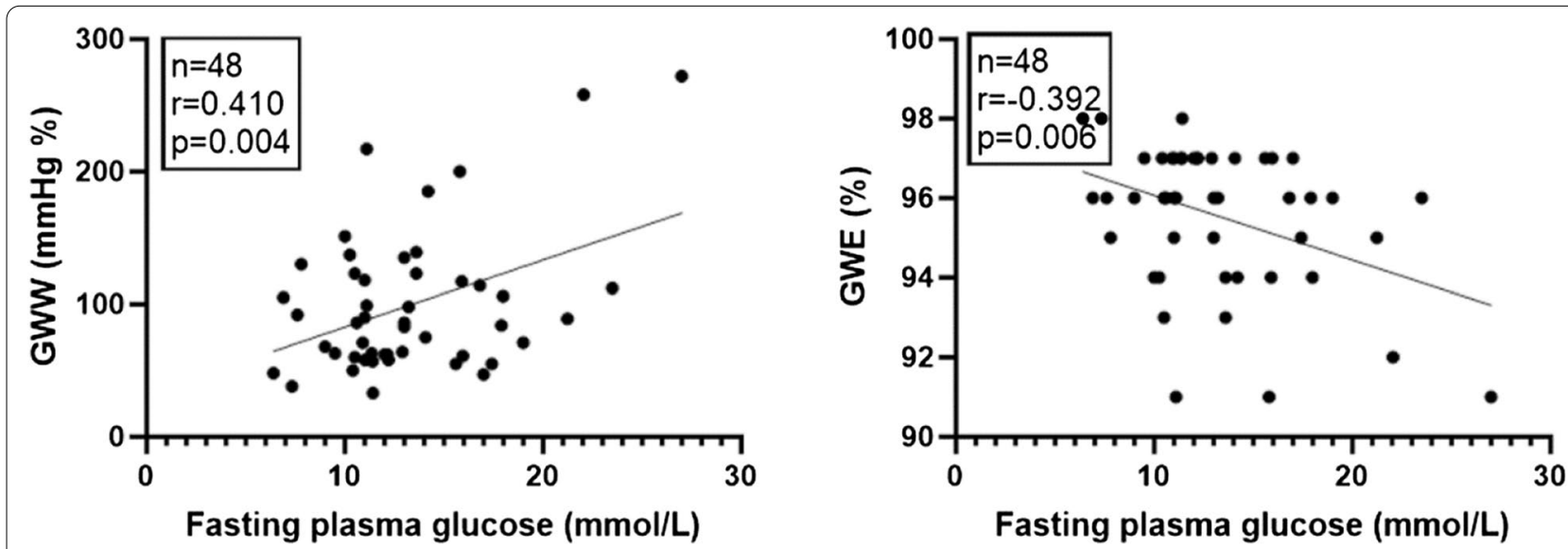

Fig. 4 Correlation tests showed in T2DM patients, fasting plasma glucose was positively correlate with GWW, while negatively correlate with GWE 

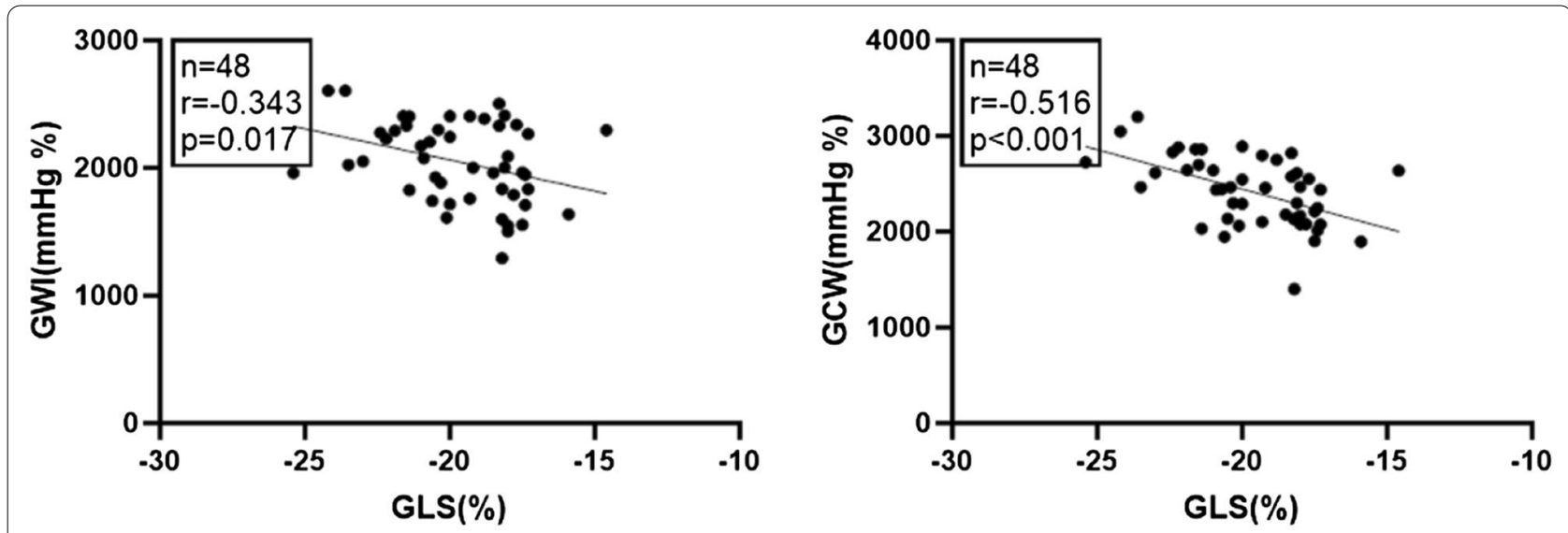

Fig. 5 Correlation tests showed in T2DM patients, GLS was negatively correlated with GWI and GCW

Table 5 ICCS for intra- and interobserver variability for GLS and MW parameters

\begin{tabular}{llllll}
\hline Variable & \multicolumn{2}{l}{$\begin{array}{l}\text { Interobserver } \\
\text { variability }\end{array}$} & & \multicolumn{2}{l}{$\begin{array}{l}\text { Intraobserver } \\
\text { variability }\end{array}$} \\
\cline { 2 - 3 } & ICC & $\mathbf{9 5 \%} \mathbf{C l}$ & & ICC & $\mathbf{9 5 \% ~ C l}$ \\
\hline GLS (\%) & 0.911 & $0.809-0.960$ & & 0.923 & $0.834-0.965$ \\
GWI (mmHg \%) & 0.926 & $0.839-0.967$ & & 0.932 & $0.851-0.969$ \\
GCW (mmHg \%) & 0.914 & $0.815-0.961$ & & 0.878 & $0.743-0.944$ \\
GWW (mmHg \%) & 0.996 & $0.991-0.998$ & & 0.987 & $0.971-0.994$ \\
GWE (\%) & 0.973 & $0.939-0.998$ & & 0.967 & $0.926-0.985$ \\
\hline
\end{tabular}

\section{Limitations}

First, the number of enrolled subjects in this study was small and it is also a single center study, in the future research, we will expand the sample size to makes the research more reliable. Second, 2D-STE has the shortcoming of the speckle "out of plane motion", if the speckles move out of plane during contraction, the software cannot be tracked successfully. Third, patients with COPD were insufficient and excluded for the analysis.

\footnotetext{
Abbreviations

LV: Left ventricle; LVEF: Left ventricle ejection fraction; T2DM: Type 2 diabetes mellitus; GLS: Global longitudinal strain; MW: Myocardial work; PSL: Pressurestrain loops; GMW: Global myocardial work; GWl: Global myocardial work index; GCW: Global constructive work; GWW: Global wasted work; GWE: Myocardial work efficiency; STE: Speckle-tracking echocardiography; TDI: Tissue Doppler imaging; HR: Heart rate; BMI: Body mass index; SBP: Systolic blood pressure; DBP: Diastolic blood pressure; TCH: Total cholesterol; TG: Triglyceride; HDL: High density lipoprotein; LDL: Low density lipoprotein; LAD: Left atrial diameter; IVSd: Interventricular septal thickness in end-diastolic period; LVPWd: Left ventricular posterior wall thickness in end-diastolic period. LVDd: LV diameter in end-diastole period; LVEDV: Left ventricular enddiastolic volume; LVESV: Left ventricular end-systolic volume; HbA1c: Glycated haemoglobin; ROC: Receiver operating characteristic; AUC: Area under the
} curve; ICCs: Intraclass correlation coefficients; CRT: Cardiac resynchronization therapy; PCI: Percutaneous coronary intervention; TAVR: Transcatheter aortic valve replacement.

\section{Acknowledgements}

We would like to thank the department of Echocardiography and radiology, the Affiliated Changzhou No. 2 People's Hospital with Nanjing Medical University.

\section{Authors' contributions}

$J H$ and TW designed the study and carried out the study, data collection and analysis, TW wrote the manuscript and JH revised the manuscript. LF designed part of the experiments, and collected the T2DM patients and normal controls. $\mathrm{LL}$ performed the statistical analysis. All authors have read and approved the final manuscript.

\section{Funding}

This study was funded by Young Talent Development Plan of Changzhou Health Commission (No.: CZQM2020061), Talent Development Plan of Changzhou No. 2 People's hospital (No.: YJRC202031) and Changzhou Science and Technique Program (Grant No.: CJ20190098).

\section{Availability of data and materials}

The datasets used and analyzed during the current study are available from the corresponding author on reasonable request.

\section{Declarations}

Ethics approval and consent to participate

The study complied with the Declaration of Helsinki and was reviewed and approved by the Human Subjects Committee of Changzhou No. 2 People's Hospital. Written informed consent was obtained from each couple enrolled in the study.

\section{Consent to publication}

This manuscript does not include any individual person's data.

\section{Competing interests}

The authors declared no conflict of interest.

\section{Author details}

${ }^{1}$ Department of Radiology, The Affiliated Changzhou No. 2 People's Hospital with Nanjing Medical University, Changzhou 213003, China. ${ }^{2}$ Department of Pediatrics, Changzhou Fourth People's Hospital, Changzhou Tumor Hospital Affiliated to Soochow University, Changzhou 213003, China. ${ }^{3}$ Department of Echocardiography, The Affiliated Changzhou No. 2 People's Hospital with Nanjing Medical University, Changzhou 213003, China. 
Received: 14 November 2021 Accepted: 29 December 2021

Published online: 28 January 2022

\section{References}

1. Low Wang CC, Hess CN, Hiatt WR, Goldfine AB. Clinical update: cardiovascular disease in diabetes mellitus: atherosclerotic cardiovascular disease and heart failure in type 2 diabetes mellitus - mechanisms, management, and clinical considerations. Circulation. 2016;133:2459-502.

2. Bugger $\mathrm{H}$, Abel ED. Molecular mechanisms of diabetic cardiomyopathy. Diabetologia. 2014;57:660-71.

3. Aneja A, Tang WH, Bansilal S, Garcia MJ, Farkouh ME. Diabetic cardiomyopathy: insights into pathogenesis, diagnostic challenges, and therapeutic options. Am J Med. 2008;121:748-57.

4. Cao Y, Zeng W, Cui Y, Kong X, Wang M, Yu J, et al. Increased myocardial extracellular volume assessed by cardiovascular magnetic resonance T1 mapping and its determinants in type 2 diabetes mellitus patients with normal myocardial systolic strain. Cardiovasc Diabetol. 2018;17:7.

5. Deepti S, Roy A, Patel CD, Tandon N, Naik N, Singh S, et al. Assessment of asymptomatic ischemic heart disease using stress myocardial perfusion imaging in patients with type 2 diabetes mellitus. Indian Heart J. 2018;70(Suppl 3):S157-60.

6. Galderisi M, de Simone G, Innelli P, Turco A, Turco S, Capaldo B, et al. Impaired inotropic response in type 2 diabetes mellitus: a strain rate imaging study. Am J Hypertens. 2007;20:548-55.

7. Ernande L, Bergerot C, Girerd N, Thibault H, Davidsen ES, Gautier PignonBlanc $P$, et al. Longitudinal myocardial strain alteration is associated with left ventricular remodeling in asymptomatic patients with type 2 diabetes mellitus. J Am Soc Echocardiogr. 2014;27:479-88.

8. Ng AC, Delgado V, Bertini M, van der Meer RW, Rijzewijk LJ, Hooi Ewe S, et al. Myocardial steatosis and biventricular strain and strain rate imaging in patients with type 2 diabetes mellitus. Circulation. 2010;122:2538-44.

9. Pedrizzetti G, Claus P, Kilner PJ, Nagel E. Principles of cardiovascular magnetic resonance feature tracking and echocardiographic speckle tracking for informed clinical use. J Cardiovasc Magn Reson. 2016;18:51.

10. Scatteia A, Baritussio A, Bucciarelli-Ducci C. Strain imaging using cardiac magnetic resonance. Heart Fail Rev. 2017;22:465-76.

11. Huang J, Hu HL, Yan ZN, Fan L, Rui YF, Shen D, et al. Peak systolic longitudinal rotation: a new tool for detecting left ventricular systolic function in patients with type 2 diabetes mellitus by two-dimensional speckle tracking echocardiography. BMC Cardiovasc Disord. 2019;19:137.

12. Alberti KG, Zimmet PZ. Definition, diagnosis and classification of diabetes mellitus and its complications. Part 1: diagnosis and classification of diabetes mellitus provisional report of a WHO consultation. Diabet Med. 1998:15:539-53.

13. Chan J, Edwards NFA, Khandheria BK, Shiino K, Sabapathy S, Anderson $B$, et al. A new approach to assess myocardial work by non-invasive left ventricular pressure-strain relations in hypertension and dilated cardiomyopathy. Eur Heart J Cardiovasc Imaging. 2019;20:31-9.

14. Edwards NFA, Scalia GM, Shiino K, Sabapathy S, Anderson B, Chamberlain $\mathrm{R}$, et al. Global myocardial work is superior to global longitudinal strain to predict significant coronary artery disease in patients with normal left ventricular function and wall motion. J Am Soc Echocardiogr. 2019:32:947-57.

15. Lee WS, Kim J. Diabetic cardiomyopathy: where we are and where we are going. Korean J Intern Med. 2017:32:404-21.

16. Billig S, Zayat R, Ebeling A, Steffen H, Nix C, Hatam N, et al. Transesophageal echocardiography in swine: evaluation of left and right ventricular structure, function and myocardial work. Int J Cardiovasc Imaging. 2020. https://doi.org/10.1007/s10554-020-02053-7.

17. Galli E, John-Matthwes B, Rousseau C, Schnell F, Leclercq C, Donal E. Echocardiographic reference ranges for myocardial work in healthy subjects: a preliminary study. Echocardiography. 2019;36:1814-24.

18. Meimoun P, Abdani S, Stracchi V, Elmkies F, Boulanger J, Botoro T, et al. Usefulness of noninvasive myocardial work to predict left ventricular recovery and acute complications after acute anterior myocardial infarction treated by percutaneous coronary intervention. J Am Soc Echocardiogr. 2020;33:1180-90
19. Fortuni F, Butcher SC, van der Kley F, Lustosa RP, Karalis I, de Weger A, et al. Left ventricular myocardial work in patients with severe aortic stenosis. J Am Soc Echocardiogr. 2020. https://doi.org/10.1016/j.echo.2020.10.014.

20. Jain R, Bajwa T, Roemer S, Huisheree H, Allaqaband SQ, Kroboth S, et al. Myocardial work assessment in severe aortic stenosis undergoing transcatheter aortic valve replacement. Eur Heart J Cardiovasc Imaging. 2020. https://doi.org/10.1093/ehjci/jeaa257.

21. Cui C, Liu L, Li Y, Liu Y, Huang D, Hu Y, et al. Left ventricular pressure-strain loop-based quantitative examination of the global and regional myocardial work of patients with dilated cardiomyopathy. Ultrasound Med Biol. 2020;46:2834-45

22. Hiemstra YL, van der Bijl P, El Mahdiui M, Bax JJ, Delgado V, Marsan NA. Myocardial work in nonobstructive hypertrophic cardiomyopathy: implications for outcome. J Am Soc Echocardiogr. 2020;33:1201-8.

23. Ke QQ, Xu HB, Bai J, Xiong L, Li MM. Evaluation of global and regional left ventricular myocardial work by echocardiography in patients with chronic kidney disease. Echocardiography. 2020. https://doi.org/10.1111/ echo.14864.

24. Clemmensen TS, Eiskjaer H, Ladefoged B, Mikkelsen F, Sorensen J, Granstam SO, et al. Prognostic implications of left ventricular myocardial work indices in cardiac amyloidosis. Eur Heart J Cardiovasc Imaging. 2020 https://doi.org/10.1093/ehjci/jeaa097.

25. Liu JH, Chen Y, Yuen M, Zhen Z, Chan CW, Lam KS, et al. Incremental prognostic value of global longitudinal strain in patients with type 2 diabetes mellitus. Cardiovasc Diabetol. 2016:15:22.

\section{Publisher's Note}

Springer Nature remains neutral with regard to jurisdictional claims in published maps and institutional affiliations.

Ready to submit your research? Choose BMC and benefit from

- fast, convenient online submission

- thorough peer review by experienced researchers in your field

- rapid publication on acceptance

- support for research data, including large and complex data types

- gold Open Access which fosters wider collaboration and increased citations

- maximum visibility for your research: over 100M website views per year

At BMC, research is always in progress.

Learn more biomedcentral.com/submissions 